\title{
Stability of switchable SmS for piezoresistive applications
}

By:Sousanis, A (Sousanis, Andreas) ${ }^{[1]}$; Smet, PF (Smet, Philippe F.) ${ }^{[1]}$; Detavernier, C (Detavernier, Christophe) ${ }^{[1]}$; Poelman, D (Poelman, Dirk) ${ }^{[1]}$

Book Group Author(s):IEEE

View ResearcherID and ORCID

2016 IEEE NANOTECHNOLOGY MATERIALS AND DEVICES CONFERENCE (NMDC)

Book Series: IEEE Nanotechnology Materials and Devices Conference

Published: 2016

\section{Conference}

Conference: 11th IEEE Nanotechnology Materials and Devices Conference (NMDC)

Location: Toulouse, FRANCE

Date: OCT 09-12, 2016

Sponsor(s):IEEE; IEEE Nanotechnol Council

\section{Abstract}

In this work samarium sulfide thin films have been deposited by using e-beam evaporation under $\mathrm{H} 2 \mathrm{~S}$ atmosphere. Optical and structural properties were studied in several environments. The stability in several environments allows further processing into piezoelectronic devices. 


\title{
Stability of switchable SmS for piezoresistive applications*
}

\author{
Andreas Sousanis, Philippe F. Smet, Christophe Detavernier, Dirk Poelman
}

$1 \square$

\begin{abstract}
In this work samarium sulfide thin films have been deposited by using e-beam evaporation under $\mathrm{H}_{2} \mathrm{~S}$ atmosphere. Optical and structural properties were studied in several environments. The stability in several environments allows further processing into piezoelectronic devices.
\end{abstract}

\section{INTRODUCTION}

$\mathrm{SmS}$ is a switchable material, providing a pressure-induced transition from the semiconducting to the metallic state. The close vicinity between the $5 \mathrm{~d}$ state and the $4 \mathrm{f}$ states of $\mathrm{Sm}$ ions is the origin of the switching behavior between the two states. The metallic state can switch back to the semiconducting state by annealing or upon release of the pressure in the case of using other rare-earths elements, such as Eu and Gd.[1] The electronic changes between the states are accompanied by changes in optical and electrical properties. By applying pressure to $\mathrm{SmS}$, the $5 \mathrm{~d}$ degenerate state moves towards the $4 \mathrm{f}$ state of the Sm ion. Practically, the pressure, which can be provided by polishing or scratching, increases the energy distance between doubly degenerate $\left(\mathrm{e}_{\mathrm{g}}\right)$ and triply degenerate $\left(\mathrm{t}_{2 \mathrm{~g}}\right)$ states. [2] In $\mathrm{SmS}$ there is no phase change, but only volume collapse during the acquisition of the metallic state. XRD patterns of both states are displayed in Fig. 1 for a $\mathrm{SmS}$ thin film with a thickness of $50 \mathrm{~nm}$. The switching behavior can be used in low-voltage consumption transistors and gauge sensors. Apart from the aforementioned transition, an additional magnetic transition from the paramagnetic state to an antiferromagneticly ordered state takes place at around 18.5 kbar at 0K. [3]

Even though the transition back to the semiconducting state reportedly takes place at $400^{\circ} \mathrm{C}$, [4] we found that the transition back to the semiconducting state initiates at lower temperatures. Nonetheless, the temperature of around $400^{\circ} \mathrm{C}$ is quite crucial as at that temperature two effects occur. The first one is related to the transition back to the

*This project has received funding from the European Union's Horizon 2020 research and innovation programme under grant agreement No 688282, PETMEM (Piezoelectronic Transduction Memory Device).

A.S. is with LumiLab, Department of Solid State Sciences, Ghent University, Krijgslaan 281-S1, 9000 Gent, Belgium (e-mail: andreas.sousanis@ugent.be),

P.F.S. is with LumiLab, Department of Solid State Sciences, Ghent University, Krijgslaan 281-S1, 9000 Gent, Belgium (corresponding author, phone:+32-9-264-4353;fax:+32-9-264-4996;email:philippe.smet@ugent.be)

C.D. is with CoCooN, Department of Solid State Sciences, Ghent University, Krijgslaan 281-S1, 9000 Gent, Belgium (e-mail: christophe.detavernier@ugent.be).

D.P is with LumiLab, Department of Solid State Sciences, Ghent University, Krijgslaan 281-S1, 9000 Gent, Belgium (e-mail: dirk.poelman@ugent.be). semiconducting state and the second one stems from the oxidation of SmS thin films at that temperature, when annealed under ambient air.

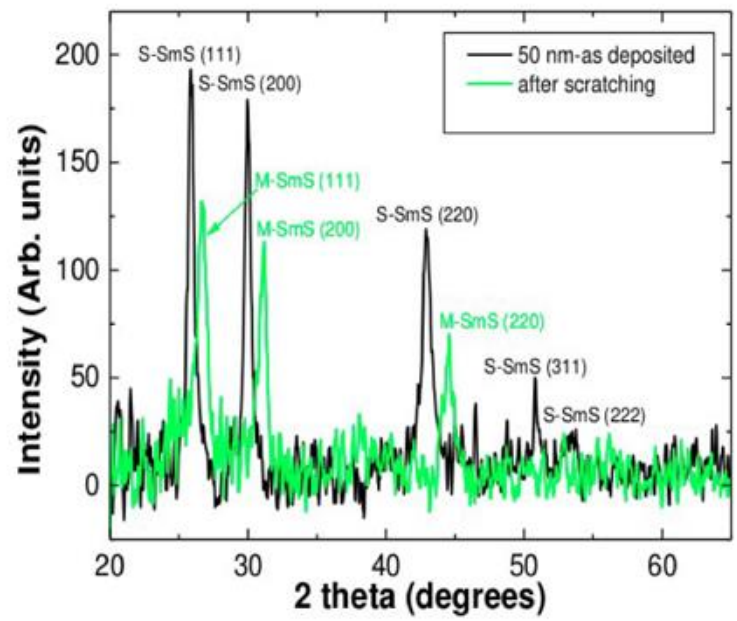

Figure 1. XRD patterns of a $50 \mathrm{~nm} \mathrm{SmS} \mathrm{thin} \mathrm{film} \mathrm{in} \mathrm{semi-conducting} \mathrm{state}$ (S-SmS), and after transition into the metallic one (M-SmS).

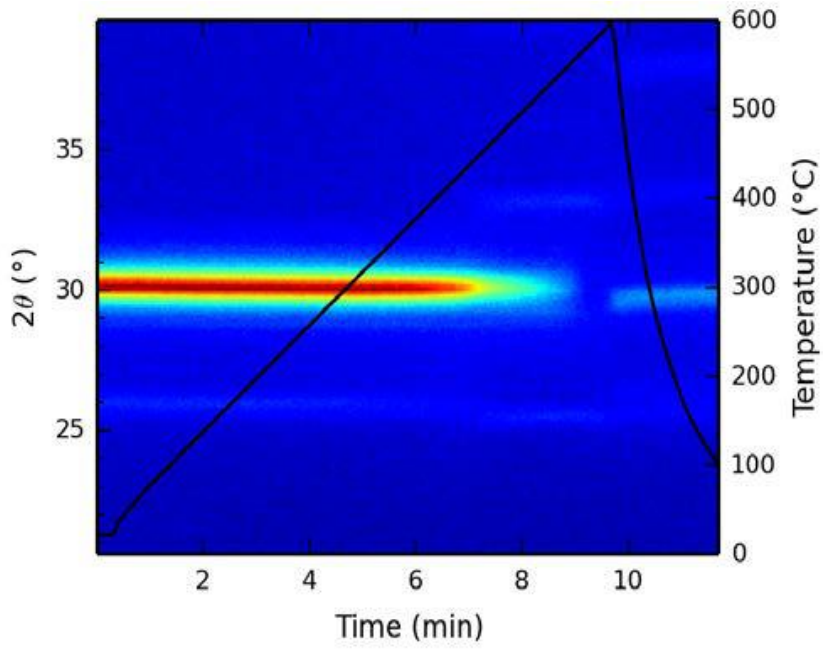

Figure 2. In-situ XRD pattern of the semiconducting state of SmS after annealing in an atmosphere containing $80 \%$ of $\mathrm{He}$ and $20 \%$ of $\mathrm{O}_{2}$. Black line represents annealing and cooling down of film as a function of time.

Optical and structural studies have shown that $\mathrm{SmS}$ is quite stable upon annealing under ambient air at $300^{\circ} \mathrm{C}$ to $350^{\circ} \mathrm{C}$, without any important traces of any other compounds which would strongly modulate the switching behavior. Insitu XRD measurements (Fig. 2) showed that $\mathrm{SmS}$ is stable up to $350^{\circ} \mathrm{C}$ when annealing in a mixed oxygen-helium atmosphere. Further increase in temperature leads to the formation of other crystalline phases. Finally, the behavior of $\mathrm{SmS}$ films under $\mathrm{N}_{2}$ and $\mathrm{H}_{2} \mathrm{~S}$ annealing will also be presented. 
For the thin film deposition of $\mathrm{SmS}$, we used e-beam evaporation of metallic $\mathrm{Sm}$ in $\mathrm{H}_{2} \mathrm{~S}$ atmosphere. Structural characterization of the S-SmS thin films was carried out by X-ray diffraction (XRD) using a standard powder diffractometer (Siemens D8) with CuKa1 radiation $\lambda=0.154059 \mathrm{~nm})$. Selected samples were viewed via scanning electron microscopy (SEM). The SEM analysis was carried out in a FEI electron microscope, with a point resolution of $1.7 \mathrm{~nm}$ at $20 \mathrm{kV}$. The Uv-Vis. spectra were recorded at room temperature in transmission geometry with a Varian Cary 500 Uv-Vis. spectrophotometer in the wavelength range of 200-1100 $\mathrm{nm}$.

\section{II.CONCLUSION}

Our results demonstrate that $\mathrm{SmS}$ can be used in several environments, allowing a wide range of processing in microelectronics industry. Therefore, possible usage of SmS on low-voltage high-speed piezoelectronic memory devices [2,5] becomes reality, as the close vicinity of the $5 \mathrm{~d}$ band with the $4 \mathrm{f}$ states of $\mathrm{Sm}^{2+}$ (switching behavior) provides suitable characteristics for future devices, with quite low energy consumption in comparison with conventional memory devices (e.g. flash devices).

\section{REFERENCES}

[1] E. Rogers, P. F. Smet, P. Dorenbos, D. Poelman and E. van der Kolk, "The thermally induced metal-semiconducting phase transition of samarium monosulfide (SmS) thin films," J. Phys.: Condens. Matter, vol. 22, 015005 , 2010.

[2] I.-B. Magdău, X.-H. Liu, M. A. Kuroda, T. M. Shaw, J. Crain, P. M. Solomon, D. M. Newns, and G. J. Martyna, "The piezoelectronic stress transduction switch for very large-scale integration, low voltage sensor computation, and radio frequency applications," Appl. Phys. Lett., vol. 107, 073505, 2015.

[3] A. Barla, J-P Sanchez, J. Derr, B Salce, G. Lapertot, J. Flouquet, B. P. Doyle, O. Leupold, R. Ruffer M. M. Abd-Elmeguid and R. Lengsdorf, "Valence and magnetic instabilities in Sm compounds at high pressures," J. Phys.: Condens. Matter, vol. 17 pp. S837-S848, 2005.

[4] T.L. Bzhalava, S.G. Shulman, T.T. Dedegkayen, T.B. Zhukova, I.A. Smirnov, Metal modification of SmS in vacuum-deposited films," Physics Letters A, vol. 55, pp. 161-162, 1975.

[5] D. Newns, B. Elmegreen, X. H. Liu, G. Martyna, "A low-voltage high-speed electronic switch based on piezoelectric transduction," J. Appl. Phys., vol. 111, 084509, 2012. 